\title{
Phase I dose-escalation study of S-222611, an oral reversible dual tyrosine kinase inhibitor of EGFR and HER2, in patients with solid tumours
}

\section{Authors}

James Spicer ${ }^{1}$, Richard Baird ${ }^{2}$, Aneta Suder ${ }^{1}$, Nicola Cresti ${ }^{3}$, Javier Garcia Corbacho $^{2}$, Linda Hogarth ${ }^{3}$, Eugene Frenkel ${ }^{4}$, Sayaka Matsumoto ${ }^{5}$, Izumi Kawabata ${ }^{5}$, Kirsteen Donaldson ${ }^{5}$, John Posner ${ }^{5}$, Debashis Sarker ${ }^{1}$, Duncan Jodrell ${ }^{2}$ \& Ruth Plummer $^{3}$

${ }^{1}$ King's College London, Guy's Hospital, London, UK; ${ }^{2}$ University of Cambridge, Department of Oncology, Cambridge, ${ }^{3}$ Northern Centre for Cancer Care, Newcastle upon Tyne, UK; ${ }^{4}$ University of Texas Southwestern Medical Center, Dallas, TX; ${ }^{5}$ Shionogi \& Co. Ltd., Osaka, Japan

\section{Corresponding author}

James Spicer

Division of Cancer Studies

King's College London

Guy's Hospital

Great Maze Pond

London SE1 9RT, UK

james.spicer@kcl.ac.uk 


\section{Abstract}

Background: S-222611 is a reversible inhibitor of EGFR, HER2 and HER4 with preclinical activity in models expressing these proteins. We have performed a Phase I study to determine safety, maximum tolerated dose (MTD), pharmacokinetic profile (PK) and preliminary efficacy in patients with solid tumours expressing EGFR or HER2.

Methods: We conducted this first-in-patient study at 3 sites in the UK. Subjects had advanced tumours not suitable for standard treatment, expressing EGFR and/or overexpressing HER2 using immunohistochemistry, and/or with amplified HER2 using FISH. Daily oral doses of S-222611 were escalated from $100 \mathrm{mg}$ to $1600 \mathrm{mg}$. Full plasma concentration profiles for drug and metabolites were obtained. Optional paired tumour biopsies were obtained for analysis of pharmacodynamic biomarkers.

Findings: 33 patients received S-222611. It was well tolerated, and the most common toxicities, almost all mild (grade 1 or 2), were diarrhoea, fatigue, rash and nausea. Only two dose-limiting toxicities occurred (diarrhoea and rash), which resolved on interruption. Maximum tolerated dose was not reached. Plasma exposure increased with dose up to $800 \mathrm{mg}$, exceeding levels eliciting pre-clinical responses. The plasma terminal half-life was more than $24 \mathrm{~h}$, supporting once daily dosing. Responses were seen over a wide range of doses, including one complete clinical response in a patient with HER2-positive breast carcinoma previously treated with lapatinib and trastuzumab. Six partial responses were seen in EGFR- and/or HER2-positive oesophageal, breast and renal cancers, with prolonged stable disease in a further two patients (clinical benefit in $27 \%$ ). Four patients have remained on treatment for more than 12 months.

Interpretation: Continuous daily oral S-222611 is well tolerated, and has broad and significant antitumour activity. The recommended Phase 2 dose, based on PK and efficacy, is $800 \mathrm{mg} /$ day. 


\section{Introduction}

Dysregulation of signaling via the ERBB receptor tyrosine kinase family is one of the most common oncogenic drivers in epithelial tumours (1). The members of this family, epidermal growth factor receptor (EGFR; ERBB1) and human epidermal growth factor receptors 2-4 (HER2-4), interact with each other through homo- and heterodimerization, leading to activation of their intracellular tyrosine kinase domains. Small molecule reversible inhibitors targeting EGFR are most active in tumours driven by activating EGFR mutations $(2,3)$. However, in most other epithelial tumours the complexity of signaling mediated by the various ERBB receptors predicts that specific inhibition of a single family member may be insufficient to gain sustained control of neoplastic proliferation (1). A second generation of ERBB inhibitors has been developed with broader specificity, but these have modest activity in all but a narrow range of diseases $(4,5)$. Earlier generations of ERBB inhibitors are also associated with significant on-target toxicities, especially rash and diarrhoea, at active doses, and with fatigue, which can be problematic with chronic treatment (6). New, more potent, broad spectrum, less toxic ERBB inhibitors are required.

S-222611, an oral agent, is a novel, reversible, selective and potent inhibitor of EGFR, HER2 and HER4 kinases. S-222611 inhibits the ERBB kinases with $\mathrm{IC}_{50}$ values for EGFR, HER2 and HER4 of $1.5 \mathrm{nM}, 7.2 \mathrm{nM}$ and $2.5 \mathrm{nM}$, respectively. In pre-clinical studies, S-222611 has activity against EGFR- and HER2-expressing human cell lines and xenografts, including a model of breast cancer brain and bone metastases (manuscript in preparation). In healthy male volunteers, single doses up to $400 \mathrm{mg}$ (the maximum tested) were well tolerated with an adverse event profile indistinguishable from placebo. Systemic exposure $\left(C_{\max }\right.$ and $\left.A U C_{0 \text {-inf }}\right)$ was proportional to dose and mean $\mathrm{t}_{1 / 2 \lambda z}$ ranged from 20.8 to $25.6 \mathrm{~h}$, independent of dose. Following administration of a $100 \mathrm{mg}$ tablet, $\mathrm{C}_{\max }$ and $\mathrm{AUC}_{0-\mathrm{nf}}$ were $12 \%$ and $30 \%$ 
higher in the fed than in the fasted state, respectively. The data support once daily dosing without an important effect of the time of dosing relative to meals.

These encouraging data and the unmet need for more effective and better tolerated ERBB inhibition led us to conduct a Phase I dose-escalation study of S-222611 in patients with advanced solid tumours expressing EGFR and/or HER2. The primary objectives were to evaluate safety and define the MTD of S-222611 when administered in a continuous, once-daily dosing schedule. Secondary objectives included pharmacokinetic (PK) evaluation of S-222611, preliminary assessment of anti-tumour efficacy, and assessment of pharmacodynamic markers in tumour biopsies.

\section{Patients and Methods}

\section{Study population and drug administration}

This was a Phase I, open-label, dose-escalation study of continuous once-daily oral treatment with S-222611 conducted at three centres from July 2010 to November 2012. Eligible patients had histologically confirmed solid tumours not amenable to established treatments, expressing EGFR (1+ to 3+) and/or overexpressing HER2 using immunohistochemistry, and/or with amplified HER2 using $\mathrm{FISH}(3+$ or $2+$ with amplification). Colorectal cancer patients with KRAS mutation were excluded. Other eligibility criteria included written informed consent; age $\geq 18$ years; Eastern Cooperative Oncology Group (ECOG) performance status $0-1$; completion of previous anticancer therapy at least 4 weeks before study entry with recovery from all previous therapy-related toxicities to Common Terminology Criteria for Adverse Events v4.0 (CTCAE) Grade $\leq 1$; adequate bone marrow, renal, hepatic and left ventricular function; and no concomitant anticancer or investigational drug. Patients were excluded if they had active autoimmune or infectious disease; a gastrointestinal disorder that could interfere with the absorption of oral study drug; needed treatment 
with a strong CYP3A4 inhibitor or inducer; had uncontrolled brain metastasis; were sexually active and unwilling to use effective methods of contraception; pregnant or breast feeding. The study was approved after review by the relevant regulatory and independent ethics committees, and conducted in accordance with the Declaration of Helsinki and International Conference on Harmonization (ICH) Good Clinical Practice (GCP).

S-222611 (Shionogi \& Co. Ltd, Osaka, Japan) was administered as a single dose on Day 1 with continuous daily dosing from Day 8. All doses were administered as tablets containing 100mg S-222611, within 30 minutes of eating breakfast. Based on the concentrations demonstrating efficacy in preclinical tumour models and toxicity studies, and the tolerability and pharmacokinetics obtained with single doses in healthy volunteers, a continuous daily dose of $100 \mathrm{mg}$ was selected as the starting dose for escalation in patients with solid tumours. Subsequent dose levels planned were $200,400,800,1200$ and $1600 \mathrm{mg}$ daily. Dose reduction was allowed following dose limiting toxicity (DLT), provided the toxicity had improved to less than Grade 1 and the dose interruption did not exceed 14 days. Treatment continued until disease progression, unacceptable toxicity, or withdrawal of patient's consent.

Dose escalation followed a $3+3$ design with cohort expansion to six patients if one DLT was reported during the first 28-day cycle. The MTD was defined as the highest dose at which DLT occurred in no more than one of 6 patients during the first cycle. The protocol required that if two DLTs were observed at any dose level, dose escalation was to stop and the preceding cohort expanded to include at least six patients. Safety and PK data were reviewed regularly by a data monitoring committee, which made recommendations regarding dose selection. After the dose had been escalated to $1600 \mathrm{mg}$, all cohorts receiving doses of $\geq 400 \mathrm{mg}$ were expanded to 6 patients to obtain additional PK data. 


\section{Study procedures}

Screening tests at baseline included physical examination, computerized tomography with measurement of target and non-target lesions using Response Evaluation Criteria in Solid Tumours (RECIST) version1.1, complete blood count, clotting, tests of renal and liver function, electrocardiogram (ECG), left ventricular ejection fraction (LVEF) by echocardiogram or multiple-gated acquisition (MUGA) scan, and ophthalmic examination because of corneal opacification observed in preclinical toxicity studies. ECG telemetry was performed for $8 \mathrm{~h}$ following the first dose. All adverse events and laboratory variables were assessed using the CTCAE version 4.0. LVEF was evaluated after 4 and 8 weeks, and subsequently at intervals of 8 weeks. Ophthalmic examination was repeated after 4 weeks, and subsequently if clinically indicated. Other safety assessments were repeated at baseline, on Days 2, $3,5,8,15$, and 22 , at weekly intervals for a further 5 weeks, and then every 2 weeks.

\section{Pharmacokinetic analysis}

Blood was drawn for measurement of plasma concentrations of S-222611 and its active dealkylated and lactam metabolites before and 2, 4, 6, 12, 24, 48 and $96 \mathrm{~h}$ after the first single dose on Day 1 , and directly before daily drug administration on Days 8, 15 and 22. An additional 24h PK profile was performed after 21 days of repeat dosing, with samples collected before and 2, 4, 6, 12 and $24 \mathrm{~h}$ after drug administration.

Plasma concentrations of S-222611 and metabolites were analyzed by a validated liquid chromatography tandem mass spectrometry (LC-MS/MS) method (Charles River Laboratories). The quantification range for S-222611 was $0.5-5.0 \mathrm{ng} / \mathrm{mL}$ (undiluted plasma). Assay performance was assessed by back-calculation of calibration standards, tabulation of the standard curve fit function parameters, and measurement of quality control samples. Standard non-compartmental analysis was 
performed to estimate the PK parameters for S-222611 and metabolites based on plasma concentration (WinNonlin® Professional 5.2 or SAS® Version 9.2).

The following PK parameters were estimated: maximum observed plasma concentration $\left(\mathrm{C}_{\max }\right)$, time from dosing to $\mathrm{C}_{\max }\left(\mathrm{T}_{\max }\right)$, area under the plasma concentration versus time curve from time zero extrapolated to infinity $\left(A \cup C_{0 \text {-inf }}\right)$, apparent total body clearance (CL/F), apparent volume of distribution (Vz/F) and terminal half-life $\left(\mathrm{t}_{1 / 2 \lambda z}\right)$ for single dose; $\mathrm{C}_{\max }, \mathrm{T}_{\max }$, area under the plasma concentration versus time curve during a dosing interval $\left(A \cup C_{T}\right)$, average plasma concentration during a dosing interval $\left(C_{a v}\right)$, and fluctuation index $\left(\left(C_{\max }-C_{\min }\right) / C_{a v}\right)$ (FI) over a dosing interval for repeat dosing.

\section{Tumour evaluation and pharmacodynamic assessment}

Tumour response was assessed according to RECIST version 1.1 (7) at intervals of 8 weeks. Efficacy parameters included complete response $(C R)$, partial response (PR), duration of response (time from $\mathrm{CR}$ or $\mathrm{PR}$ to disease progression), and progression-free survival (PFS; time from first treatment to disease progression, death, or censoring date). Tumour biopsies were obtained, where accessible, for analysis of ERBB signaling components at baseline and after 1 month of treatment.

\section{Role of the funding source}

This study was funded by the study sponsor (Shionogi \& Co., Ltd) and monitored by a contract research organisation (Quintiles). The contract research organisation collated all data and the authors performed the data analysis. The report was written by JS, with contribution from all authors, who included employees of Shionogi \& Co., Ltd. The corresponding author had full access to all the data in the study and had final responsibility for the decision to submit for publication. 


\section{Results}

A total of 33 patients were included in the dose escalation; all contributed to the safety analysis. Daily dose levels studied were $100 \mathrm{mg}(n=5), 200 \mathrm{mg}(n=3), 400 \mathrm{mg}$ $(n=6)$, 800mg $(n=6), 1200 m g(n=6)$, and 1600mg $(n=7)$. Patient characteristics are shown in Table 1.

\section{Safety and tolerability}

Of 33 patients, 29 completed the single dose and first 3 weeks of repeat dosing, and were evaluable for dose-limiting toxicity. Five patients discontinued early, due to disease progression or clinical deterioration $(n=4)$ or withdrawn consent $(n=1)$. All 33 $(100 \%)$ patients experienced at least one adverse event (AE), irrespective of relationship to study drug. Table 2 summarizes all treatment-related AEs of any grade occurring in 3 or more patients, and all Grade 3 treatment-related AEs. There were no Grade 4 toxicities or treatment-related deaths.

In general, S-222611 was very well tolerated. The majority of AEs were Grade 1/2, and no significant hematological or biochemical toxicity was observed. The most frequently reported toxicities were diarrhoea ( $n=26,79 \% ; 70 \%$ Grade $1 / 2)$, fatigue $(n=20,61 \%)$, rash $(n=19,58 \%)$, and nausea $(n=17,52 \%)$. Diarrhoea was generally self-limiting or effectively controlled with loperamide. Although Grade 3 diarrhoea did occur at or above $1200 \mathrm{mg}$, this responded to dose interruption and did not recur on re-challenge with dose reduction. Fatigue was commonly reported, but there was no trend with dose in its frequency or severity. Grade 1/2 skin disorders of all types including rash (not otherwise specified), dry skin, dermatitis acneiform, pruritus, skin chapping, and palmar-plantar erythrodysaesesthia $(n=3)$, were common; however, no Grade 3/4 acneiform or other rash was seen. Nausea, decreased appetite and vomiting were also reported frequently but without a clear relation to dose. No clinically significant LVEF decline or evidence of ophthalmic toxicity was observed. 
In 10 patients $(30 \%)$ treatment with S-222611 was associated with unconjugated hyperbilirubinaemia, Grade 1 or 2 in all but one case, unaccompanied by abnormalities of other liver function or evidence of hemolysis. Analysis of the germline UGT1A1 locus in one of these patients revealed the heterozygous ${ }^{*} 1 /{ }^{*} 28$ genotype, which is not associated with a Gilbert's syndrome phenotype as an explanation for unconjugated hyperbilirubinaemia (8). This hyperbilirubinaemia was generally self-limiting and did not result in discontinuation of dosing.

Two DLTs occurred during the first 3 weeks of daily treatment, one each at dose levels of 1200 \& $1600 \mathrm{mg}$. At 1200mg, a 77-year-old woman with HER2-positive breast cancer developed intolerable Grade 2 rash. At $1600 \mathrm{mg}$, a 38-year-old woman with non-small cell lung cancer developed diarrhoea (Grade 3) despite symptomatic treatment. Both DLTs resolved promptly on dose interruption and, after re-challenge at a reduced dose of $400 \mathrm{mg}$ daily, the patients tolerated treatment well. Although no more than one DLT was reported in any cohort, dose escalation beyond 1600mg was not undertaken, due to acquired safety and pharmacokinetic data. These data contributed to the selection of a dose of $800 \mathrm{mg}$ for evaluation in further trials.

\section{Pharmacokinetic analysis}

The PK parameters after single and repeat doses over the dose range studied are shown in Table 3. After single doses of $800 \mathrm{mg}$, geometric mean values of $\mathrm{C}_{\max }$ were greater than expected from the doubling of dose from $400 \mathrm{mg}$, but average concentrations $\left(\mathrm{C}_{\mathrm{av}}\right)$ and $\mathrm{AUC}$ over the $24 \mathrm{~h}$ dosing interval $\left(\mathrm{AUC}_{\mathrm{O}_{\mathrm{\tau}}}\right)$ at steady state were approximately dose-proportional. Exposure at $1200 \mathrm{mg}$ was similar to that at $800 \mathrm{mg}$, and at $1600 \mathrm{mg}$ was less than that at $800 \mathrm{mg}$ (Figure 1). Median $\mathrm{T}_{\max }$ values ranged from 4 to $6 \mathrm{~h}$ and were independent of dose. The half-life was very consistent over the dose range studied, with geometric mean values ranging from 31 to $36 \mathrm{~h}$. There was no consistent association of drug clearance parameters with weight or 
surface area (not shown). Based on trough levels, steady state was achieved within 7 days of repeat dosing.

Concentrations of the pharmacologically active dealkylated and lactam metabolites of S-222611 reflected those of the parent molecule, with AUC values less than $14 \%$ and $8 \%$ (molar basis), respectively, and average half-lives of $45 \mathrm{~h}$ and $25 \mathrm{~h}$, respectively.

\section{Antitumour activity}

There were two partial responses (PRs), confirmed on repeat scans two months apart, one in a patient with EGFR-positive renal cell carcinoma (RCC) treated at 200mg, who had previously been treated with sunitinib and everolimus (Figure 2a, b); and the other in a patient with EGFR-positive oesophageal carcinoma at 400mg. A complete clinical response $(\mathrm{CR})$ was observed in a patient with cutaneous HER2positive breast carcinoma, previously treated with lapatinib and trastuzumab, treated at $1200 \mathrm{mg} \mathrm{S}-222611$ (Figure 2c, d). Four unconfirmed PRs were seen in patients with HER2-positive breast cancer who had previously failed treatment with both trastuzumab and lapatinib, and in HER2- and/or EGFR-positive oesophageal carcinoma. Two patients had responses continuing beyond 18 months, and a further two (with pancreatic and vaginal adenoid cystic carcinomas) had stable disease for more than 18 months (Figure 3). Overall, clinical response or radiological tumour shrinkage of $\geq 30 \%$ was observed in 7 patients $(7 / 33 ; 21 \%$; Figure 3$)$. Disease control rate $(\mathrm{CR}+\mathrm{PR}+\mathrm{SD})$ was $27 \%$ (Figure 3 ).

\section{Pharmacodynamic analysis}

Sequential tumour biopsies, collected at baseline and on treatment, were available for phosphoprotein analysis (pEGFR, pHER2 and pHER3) from 4 patients (2 responses, 2 disease progression). Baseline pHER3 expression was high (3+) in only one patient (with HER2-amplified breast cancer), and on repeat biopsy after 
exposure to $1200 \mathrm{mg} \mathrm{S}-222611$ this expression fell $(1+)$, and the patient underwent a complete clinical response (Figure 2e, f).

\section{Discussion}

Several drugs targeting ERBB receptors have received regulatory approval, including both small molecules (gefitinib, erlotinib, lapatinib) and antibodies (trastuzumab, adotrastuzumab, pertuzumab, emtansine, cetuximab, panitumumab). This trial was conducted because the greater potency and broader specificity of S-222611 suggest a potential for improvement in clinical efficacy compared with these earliergeneration drugs. Some patients seem to benefit from treatment with novel, improved ERBB receptor inhibitors despite disease progression on established ERBB blockers, suggesting continued dependence on, or addiction to, signaling via this pathway in these cancers (9).

We describe the dose escalation Phase 1 trial of oral S-222611, a reversible inhibitor of EGFR, HER2 and HER4, administered daily to patients with tumours overexpressing EGFR or HER2. This drug was very well tolerated, with good pharmacokinetic properties, and we observed durable efficacy in several tumour types, including patients with breast, oesophageal and renal cancers. These results support further development of this small molecule in patients with cancers expressing relevant biomarkers.

The most frequent toxicities seen with S-222611 were diarrhoea, fatigue, rash and nausea, with few of Grade 3 and none of Grade 4 severity. Although an MTD was not reached, DLTs of rash in one patient, and diarrhoea in another, were observed in separate cohorts. The toxicities of S-222611 are consistent in nature, but not in frequency or severity, with those of first generation ERBB-specific inhibitors $(5,10$ 12). At doses of S-222611 associated with clinical activity, rash requiring systemic antibiotics or topical steroids was not seen, and diarrhoea was manageable with 
symptomatic treatment without dose reduction. This compares favorably with second generation pan-HER inhibitors, which have been associated with rates of grade 3-4 diarrhoea up to $30 \%$, leading to treatment discontinuation in $6-14 \%$ of patients $(13$, 14). No significant decline in left ventricular ejection fraction occurred despite the known expression of HER2 on cardiac myocytes (15). Observed efficacy, pharmacodynamic and pharmacokinetic data informed a selection of $800 \mathrm{mg}$ administered once daily as the recommended dose for Phase 2 study. The tolerability of prolonged S-222611 dosing at clinically effective doses, particularly with respect to fatigue and to the on-target EGFR-mediated toxicities of rash and diarrhoea, represent a contrast with first generation drugs.

PK analysis suggested an approximately dose-proportional relationship up to a dose of $800 \mathrm{mg}$ daily. PK data suggest that above this dose absorption becomes saturated. Trough S-222611 concentrations at steady state with daily doses of $400 \mathrm{mg}$ or greater exceeded those that inhibit EGFR and HER2 in vitro (manuscript in preparation). All PK parameters displayed relatively high inter-patient variability, as seen with other ERBB-targeted tyrosine kinase inhibitors, including erlotinib, gefitinib, lapatinib, and afatinib (4, 5, 10-12). An unconjugated hyperbilirubinemia observed in a significant minority of patients appears not to be caused by UGT1A1 genotypes associated with loss of bilrubin conjugation activity (8); it is possible that S-222611 competes with bilirubin for hepatocyte transporter-mediated influx. The terminal elimination half-life of S-222611 is consistent with once-daily dosing. The association of drug clearance parameters with weight and surface area was poor, supporting the use of fixed drug dose administration.

We observed radiological shrinkage $>30 \%$ (confirmed and unconfirmed), or clinical complete response, in $21 \%(7 / 33)$ of all patients treated at any dose in this trial, including patients with HER2-positive breast cancer previously exposed to HER2targeting drugs, and patients with HER2-positive or EGFR-expressing oesophageal 
cancer. A marked and sustained response was observed in a case of multiplypretreated RCC expressing EGFR. No cases of NSCLC with known activating tyrosine kinase domain mutations were included in this trial. Stable disease for at least 6 months occurred in a further two patients, and in total 4 patients remained on study drug beyond 12 months. The clinical benefit rate (response or sustained stable disease) was $27 \%$, including unconfirmed responses. This was despite the fact that $30 \%$ of patients in this trial, including some of those deriving significant clinical benefit from S-222611, had previously been exposed to ERBB-targeting therapies.

The potency and broad specificity of S-222611, potentially providing effective blockade of all possible signaling-active ERBB family dimers, may explain the activity of this drug, especially in patients previously treated with early-generation ERBBtargeting drugs (whether small molecules or antibodies). HER3 has impaired kinase activity, undergoing phosphorylation only when it is heterodimerized with another ERBB receptor (16). Thus levels of pHER3 provide an indication of the effectiveness of inhibition across this receptor family. HER3 appears to play a particularly key role in HER2-positive breast cancer (17). In this trial a complete clinical response occurred in a patient with HER2-amplified breast cancer, coincident with a marked reduction in pHER3 expression.

S-222611 is very well tolerated when administered orally, once daily, even for long periods of continuous dosing. $800 \mathrm{mg}$ was established as the recommended Phase 2 dose, with promising antitumour activity in a variety of tumour types expressing receptors targeted by this inhibitor. Further study evaluating the efficacy of S-222611 is in progress. 


\section{Research in context}

\section{Systematic review:}

We searched PubMed and the American Society of Clinical Oncology virtual meeting database for reports published in English between January 1, 2003, and September 1, 2013, with the terms "EGFR neu receptors", "receptor, Epidermal Growth Factor", "receptor, erbB-2", "receptor tyrosine-protein kinase, erbB-4", "antagonists and inhibitors", and the names of several tyrosine kinase inhibitors in clinical development (e.g. afatinib, dacomitinib, neratinib). These agents reversibly or irreversibly inhibit the tyrosine kinase domain various members of the ERBB receptor superfamily (1). Early trials with drugs in this class did not select patients for target expression, but efficacy is improved by targeting only those with tumours driven by mutation or overexpression of ERBB receptors $(2,3,5,9)$.

\section{Interpretation:}

We have conducted a Phase I dose-escalation study of S-222611, the first use of this novel inhibitor of EGFR, HER2 and HER4 in cancer patients. There is unmet need for new inhibitors of these targets, with increased potency, broader specificity, and better-tolerated chronic administration, especially with chronic administration, because of the limited efficacy of early-generation therapies in this class. Eligible patients had evidence of upregulated tumour ERBB signaling. S-222611 appears safe, and associated with manageable toxicities even after chronic dosing. Significant efficacy has been demonstrated, and further study of this new drug is underway. 


\section{Acknowledgements and grant support}

The authors acknowledge financial support from the UK Department of Health via the National Institute for Health Research (NIHR) Biomedical Research Centre (BRC) award to Guy's \& St Thomas' NHS Foundation Trust in partnership with King's College London and King's College Hospital NHS Foundation Trust (and NIHR Clinical Research Facility), and to The University of Cambridge and Cambridge University Hospital NHS Foundation Trust. Cambridge, King's College London, and Newcastle are Experimental Cancer Medicine Centres. We thank Tony Marinaki for the UGT1A1 genotyping. The authors particularly thank the patients, their families and the clinical research teams for their contribution to this study.

\section{References}

1. Yarden $Y$, Pines G. The ERBB network: at last, cancer therapy meets systems biology. Nat Rev Cancer 2012;12: 553-63.

2. Rosell R, Carcereny E, Gervais R, et al. Erlotinib versus standard chemotherapy as first-line treatment for European patients with advanced EGFR mutation-positive non-small-cell lung cancer (EURTAC): a multicentre, open-label, randomised phase 3 trial. Lancet Oncol 2012;13: 239-46.

3. Mok TS, Wu YL, Thongprasert S, et al. Gefitinib or carboplatin-paclitaxel in pulmonary adenocarcinoma. N Engl J Med 2009;361: 947-57.

4. Yap TA, Vidal L, Adam J, et al. Phase I trial of the irreversible EGFR and HER2 kinase inhibitor BIBW 2992 in patients with advanced solid tumours. J Clin Oncol 2010;28: 3965-72.

5. Burris HA, 3rd, Hurwitz HI, Dees EC, et al. Phase I safety, pharmacokinetics, and clinical activity study of lapatinib (GW572016), a reversible dual inhibitor of epidermal growth factor receptor tyrosine kinases, in heavily pretreated patients with metastatic carcinomas. J Clin Oncol 2005;23: 5305-13. 
6. Postel-Vinay S, Gomez-Roca, C., Molife, L.R., et al. Phase I trials of molecularly targeted agents: should we pay more attention to late toxicities? J Clin Oncol 2011;29: 1728-35.

7. Eisenhauer EA, Therasse $\mathrm{P}$, Bogaerts $\mathrm{J}$, et al. New response evaluation criteria in solid tumours: revised RECIST guideline (version 1.1). Eur $\mathrm{J}$ Cancer 2009;45: 228-47.

8. Kadakol A, Ghosh SS, Sappal BS, Sharma G, Chowdhury JR, Chowdhury NR. Genetic lesions of bilirubin uridine-diphosphoglucuronate glucuronosyltransferase (UGT1A1) causing Crigler-Najjar and Gilbert syndromes: correlation of genotype to phenotype. Hum Mutat 2000;16: 297-306.

9. Miller VA, Hirsh V, Cadranel J, et al. Afatinib versus placebo for patients with advanced, metastatic non-small-cell lung cancer after failure of erlotinib, gefitinib, or both, and one or two lines of chemotherapy (LUX-Lung 1): a phase $2 b / 3$ randomised trial. Lancet Oncol 2012;13: 528-38.

10. Hidalgo M, Siu LL, Nemunaitis J, et al. Phase I and pharmacologic study of OSI-774, an epidermal growth factor receptor tyrosine kinase inhibitor, in patients with advanced solid malignancies. J Clin Oncol 2001;19: 3267-79.

11. Herbst RS, Maddox AM, Rothenberg ML, et al. Selective oral epidermal growth factor receptor tyrosine kinase inhibitor ZD1839 is generally well-tolerated and has activity in non-small-cell lung cancer and other solid tumours: results of a phase I trial. J Clin Oncol 2002;20: 3815-25.

12. Ranson M, Hammond LA, Ferry D, et al. ZD1839, a selective oral epidermal growth factor receptor-tyrosine kinase inhibitor, is well tolerated and active in patients with solid, malignant tumours: results of a phase I trial. J Clin Oncol 2002;20: 224050 .

13. Burstein HJ, Sun, Y., Dirix, L.Y., et al. Neratinib, an irreversible ErbB receptor tyrosine kinase inhibitor, in patients with advanced ErbB2-positive breast cancer. $\mathrm{J}$ Clin Oncol 2010;28: 1301-7. 
14. Wong K-K, Fracasso, P.M., Bukowski, R.M., et al. A phase I study with neratinib (HKI-272), an irreversible pan ErbB receptor tyrosine kinase inhibitor, in patients with solid tumours. Clin Cancer Res 2009;15: 2552-8.

15. Negro A, Brar BK, Lee KF. Essential roles of Her2/erbB2 in cardiac development and function. Recent Prog Horm Res 2004;59: 1-12.

16. Kim HH, Vijapurkar, U., Hellyer, N. J., et al. Signal transduction by epidermal growth factor and heregulin via the kinase-deficient ErbB3 protein. Biochem $\mathrm{J}$ 1998;334: 189-95.

17. Lee-Hoeflich ST, Crocker, L., Yao, E., et al. A central role for HER3 in HER2- amplified breast cancer: implications for targeted therapy. Cancer Res 2008;68: 5878-87. 


\section{List of tables:}

Table 1. Patient demographics - all treated patients $(n=33)$.

Table 2. Treatment-related adverse events (AEs): all AEs reported by 3 or more patients, and all related Grade 3 AEs.

Table 3. Geometric mean (Geometric CV \%) pharmacokinetic parameters of S222611 on Day 1 after single oral administration of 100, 200, 400, 800, 1200 and 1600mg S-222611 tablets, and at Day 28 (steady state) after 21-day continuous dosing once daily. 
Table 1 Patient demographics - all treated patients $(n=33)$

\begin{tabular}{|c|c|c|}
\hline \multirow[b]{2}{*}{ Sex } & \multicolumn{2}{|c|}{ n (\%) } \\
\hline & & \\
\hline Male & 24 & $(72.7 \%)$ \\
\hline Female & 9 & $(27.3 \%)$ \\
\hline Median age, years (range) & 63 & $(24-79)$ \\
\hline \multicolumn{3}{|l|}{ Primary cancer } \\
\hline Oesophageal/gastric & 7 & $(21.2 \%)$ \\
\hline Non-small cell lung & 5 & $(15.2 \%)$ \\
\hline Pancreatic & 5 & $(15.2 \%)$ \\
\hline Renal & 4 & $(12.1 \%)$ \\
\hline Breast & 3 & $(9.1 \%)$ \\
\hline Other & 9 & $(27.3 \%)$ \\
\hline \multicolumn{3}{|l|}{ ECOG PS at screening } \\
\hline 0 & 8 & $(24.2 \%)$ \\
\hline 1 & 25 & $(75.8 \%)$ \\
\hline \multicolumn{3}{|l|}{ All prior therapies } \\
\hline $1-2$ & 27 & $(81.8 \%)$ \\
\hline$>3$ & 6 & $(18.2 \%)$ \\
\hline \multicolumn{3}{|l|}{ Prior ErbB-targeted therapies } \\
\hline Yes & 10 & $(30.3 \%)$ \\
\hline No & 23 & $(69.7 \%)$ \\
\hline \multicolumn{3}{|l|}{ Prior chemotherapy } \\
\hline 0 & 3 & $(9.1 \%)$ \\
\hline $1-2$ & 29 & $(87.9 \%)$ \\
\hline$>3$ & 1 & $(3.0 \%)$ \\
\hline
\end{tabular}


Table 2 Treatment-related adverse events (AEs) : AEs reported in 3 or more patients, and all related Grade 3 AEs

Values shown represent the number (\%) of patients; there were no Grade 4 or 5 treatment-related AEs

\begin{tabular}{|c|c|c|c|c|c|c|c|c|c|c|c|c|c|c|}
\hline & \multicolumn{2}{|c|}{$\begin{array}{c}100 \mathrm{mg} / \text { day } \\
\mathrm{n}=5\end{array}$} & \multicolumn{2}{|c|}{$\underset{n=3}{200 \mathrm{mg} / \text { day }}$} & \multicolumn{2}{|c|}{$\underset{n=6}{400 \mathrm{mg} / \text { day }}$} & \multicolumn{2}{|c|}{$\begin{array}{c}800 \mathrm{mg} / \text { day } \\
\mathrm{n}=6\end{array}$} & \multicolumn{2}{|c|}{$\begin{array}{c}1200 \mathrm{mg} / \text { day } \\
\mathrm{n}=6\end{array}$} & \multicolumn{2}{|c|}{$\begin{array}{c}1600 \mathrm{mg} / \text { day } \\
\mathrm{n}=7\end{array}$} & \multicolumn{2}{|c|}{$\begin{array}{c}\text { Overall } \\
n=33\end{array}$} \\
\hline Diarrhoea & $2^{*}$ & 0 & 2 & 0 & 5 & 0 & 6 & 0 & 6 & 1 & 5 & 2 & 26 (79) & $\begin{array}{ll}3 & (9)\end{array}$ \\
\hline Fatigue & $4^{*}$ & 1 & 1 & 0 & 4 & 0 & 5 & 0 & 3 & 0 & 3 & 1 & $20(61)$ & $2 \quad(6)$ \\
\hline $\operatorname{Rash}^{\dagger}$ & 2 & 0 & 1 & 0 & 2 & 0 & 4 & 0 & 6 & 0 & 4 & 0 & $19(58)$ & 0 \\
\hline Vomiting & 1 & 0 & 1 & 0 & 2 & 0 & 2 & 0 & 2 & 0 & 1 & 0 & $9 \quad(27)$ & 0 \\
\hline $\begin{array}{l}\text { Blood bilirubin } \\
\text { increased }\end{array}$ & 0 & 0 & 0 & 0 & 3 & 0 & 1 & 0 & 1 & 0 & 3 & 1 & $8 \quad(24)$ & $1 \quad(3)$ \\
\hline $\begin{array}{l}\text { Taste } \\
\text { disturbance }^{\ddagger}\end{array}$ & 2 & 0 & 0 & 0 & 1 & 0 & 1 & 0 & 1 & 0 & 2 & 0 & $7 \quad(21)$ & 0 \\
\hline $\begin{array}{l}\text { Weight } \\
\text { decreased }\end{array}$ & 0 & 0 & 0 & 0 & 1 & 0 & 1 & 0 & 2 & 0 & 1 & 0 & $5 \quad(15)$ & 0 \\
\hline
\end{tabular}




\begin{tabular}{|c|c|c|c|c|c|c|c|c|c|c|c|c|c|c|}
\hline & \multicolumn{2}{|c|}{$100 \underset{n=5}{\mathrm{mg} / \text { day }}$} & \multicolumn{2}{|c|}{$\begin{array}{c}200 \mathrm{mg} / \text { day } \\
\mathrm{n}=3\end{array}$} & \multicolumn{2}{|c|}{$\begin{array}{c}400 \mathrm{mg} / \text { day } \\
\mathrm{n}=6\end{array}$} & \multicolumn{2}{|c|}{$\begin{array}{c}800 \mathrm{mg} / \mathrm{day} \\
\mathrm{n}=6\end{array}$} & \multicolumn{2}{|c|}{$\underset{n=6}{1200 \mathrm{mg} / \text { day }}$} & \multicolumn{2}{|c|}{$\begin{array}{c}1600 \mathrm{mg} / \text { day } \\
\mathrm{n}=7\end{array}$} & \multicolumn{2}{|c|}{$\begin{array}{c}\begin{array}{c}\text { Overall } \\
n=33\end{array} \\
\end{array}$} \\
\hline Lymphopenia & 0 & 0 & 1 & 0 & 1 & 1 & 0 & 0 & 1 & 1 & 1 & 1 & $4 \quad(12)$ & $\begin{array}{ll}3 & (9)\end{array}$ \\
\hline Dry eye & 1 & 0 & 1 & 0 & 0 & 0 & 0 & 0 & 2 & 0 & 0 & 0 & $4 \quad(12)$ & 0 \\
\hline Constipation & 0 & 0 & 1 & 0 & 0 & 0 & 0 & 0 & 2 & 0 & 1 & 0 & $4 \quad(12)$ & 0 \\
\hline $\begin{array}{l}\text { Muscular } \\
\text { weakness }\end{array}$ & 0 & 0 & 0 & 0 & 0 & 0 & 0 & 0 & 0 & 0 & 1 & 1 & $1 \quad(3)$ & (3) \\
\hline GGT increased & 0 & 0 & 0 & 0 & 1 & 1 & 0 & 0 & 0 & 0 & 0 & 0 & (3) & (3) \\
\hline
\end{tabular}

* As permitted by the protocol, one patient dose-escalated from 100 to $400 \mathrm{mg}$ daily; these AEs occurred on the higher dose

$\dagger \quad$ Rash includes rash of all types and the terms dry skin, erythema, skin chapped, dermatits acneiform, pruritus and palmar-plantar erythrodysesthesia

$\mp \quad$ Taste disturbance includes coded terms dysgeusia and ageusia

** Abdominal pain includes upper abdominal pain and lower abdominal pain 
Table 2 Geometric mean (Geometric CV \%) pharmacokinetic parameters of S-222611 on Day 1 after single oral administration of $100,200,400,800,1200$ and $1600 \mathrm{mg} \mathrm{S}-222611$ tablets, and at Day 28 (steady state) after 21-day continuous dosing once daily.

\begin{tabular}{|c|c|c|c|c|c|c|}
\hline Dose mg & 100 & 200 & 400 & 800 & 1200 & 1600 \\
\hline \multicolumn{7}{|c|}{ Single dose (Day 1 ) } \\
\hline $\mathrm{n}$ & 5 & 3 & 6 & 6 & 6 & 7 \\
\hline $\mathrm{C}_{\max } \mathrm{ng} / \mathrm{mL}$ & $75.0(35.6)$ & $63.0(62.4)$ & $240(65.5)$ & $703(32.2)$ & $897(40.7)$ & $647(57.7)$ \\
\hline $\mathrm{T}_{\max } \mathrm{h}$ & $4.0(4.0,4.1)$ & $4.0(4.0,12.0)$ & $5.1(4.0,12.1)$ & $6.0(4.0,6.0)$ & $4.1(2.0,6.0)$ & $4.1(4.0,12.3)$ \\
\hline$A \cup C_{0-\text { inf }} \mathrm{ng} \cdot \mathrm{h} / \mathrm{mL}$ & $1600(27.8)$ & $1670(40.1)$ & $5050(46.4)$ & $16400(38.8)$ & $21500(41.7)^{*}$ & $14700(54.1)$ \\
\hline $\mathrm{CL} / \mathrm{F} \mathrm{L/h}$ & $62.5(28.0)$ & $120(40.3)$ & $79.2(46.4)$ & $48.7(38.8)$ & $55.8(41.8)^{*}$ & $109(54.1)$ \\
\hline $\mathrm{Vz} / \mathrm{F} \mathrm{L}$ & $3130(26.8)$ & $6120(37.5)$ & $3520(37.2)$ & $2340(30.9)$ & $2870(42.8)^{\star}$ & $4900(48.5)$ \\
\hline $\mathrm{t}_{1 / 2, \mathrm{z}} \mathrm{h}$ & $34.8(3.1)$ & $35.4(3.4)$ & $30.8(12.6)$ & $33.4(11.0)$ & $35.7(23.8)^{\star}$ & $31.3(17.7)$ \\
\hline \multicolumn{7}{|c|}{ Steady state (Days 28-29) } \\
\hline $\mathrm{n}$ & 3 & 3 & 5 & 5 & 4 & 4 \\
\hline $\mathrm{C}_{\max } \mathrm{ng} / \mathrm{mL}$ & $88.2(13.9)$ & $112(32.3)$ & $403(16.4)$ & $725(39.8)$ & $591(51.6)$ & 778 (65.4) \\
\hline $\mathrm{T}_{\max } \mathrm{h}$ & $5.9(4.2,6.0)$ & $4.0(4.0,6.1)$ & $4.0(3.8,6.2)$ & $4.1(4.0,11.1)$ & $5.0(4.0,6.0)$ & $5.1(4.0,6.0)$ \\
\hline $\mathrm{AUC}_{\tau} \mathrm{ng} \cdot \mathrm{h} / \mathrm{mL}$ & $1300(19.1)$ & $1630(32.9)$ & 5990 (21.3) & $10600(48.6)^{\star \star}$ & $10200(54.6)$ & $12300(52.0)$ \\
\hline $\mathrm{C}_{\mathrm{av}} \mathrm{ng} / \mathrm{mL}$ & $53.6(17.1)$ & $67.8(32.7)$ & $251(21.4)$ & $443(48.5)^{\star \star}$ & $404(50.0)$ & $512(56.8)$ \\
\hline $\mathrm{FI} \%$ & $108(7.8)$ & $104(5.0)$ & $104(10.8)$ & $77.9(43.4)^{\star *}$ & $79.2(25.1)$ & $74.4(73.5)$ \\
\hline
\end{tabular}

Geometric Mean (Geometric CV\%) values except $T_{\max }$ for which median and range are shown. ${ }^{*} \mathrm{n}=5 ;{ }^{* *} \mathrm{n}=4$

Abbreviations: $\mathrm{C}_{\max }$ maximum plasma concentration; $\mathrm{T}_{\max }$ time from baseline to $\mathrm{C}_{\max } ; \mathrm{AUC}_{0 \text {-inf }}$ area under plasma concentration versus time curve from time zero extrapolated to infinity; CL/F apparent total body clearance; $\mathrm{Vz} / \mathrm{F}$ apparent volume of distribution and $\mathrm{t}_{1 / 2 \mathrm{z} z}$ terminal half-life for single dose; $A \cup \mathrm{C}_{\tau}$ area under the plasma concentration versus time curve during a dosing interval; $\mathrm{C}_{\mathrm{av}}$ average plasma concentration during a dosing interval; FI Fluctuation index over a dosing interval for repeat dose. 


\section{Figure Legends}

Figure 1. Geometric mean drug plasma concentration-time profiles of S-222611 after single- and multiple-dosing oral administration of 100, 200, 400, 800, 1200 and $1600 \mathrm{mg}$.

Figure 2. Pre- (a) and post-treatment (b) CT scans showing hilar lymphadenopathy (red circle) and pleural metastases (yellow circle) in a patient with renal cell carcinoma expressing EGFR, which had previously progressed on sunitinib and everolimus. Pre- (c) and post-treatment (d) appearance of cutaneous disease in a female with HER2-positive breast cancer who had previously failed treatment with trastuzumab and lapatinib; baseline (e) and post-cycle 1 (f) tumour biopsies from the same patient showing downregulation of pHER3.

Figure 3. Patients treated at each dose level, showing biomarker upregulation in tumour samples, time on treatment, and best response by RECIST. 